\section{PERCEPÇÃO DOCENTE SOBRE A INTEGRALIDADE NA ATENÇÃO PRIMÁRIA À SAÚDE NO PROCESSO ENSINO-APRENDIZAGEM}

\author{
Perception teachers on the comprehensiveness in Primary \\ Health Care in The teaching-learning process
}

\section{RESUMO}

Objetivo: Analisar a compreensão sobre a Integralidade na perspectiva dos docentes de enfermagem e medicina inseridos na Atenção Primária à Saúde (APS). Métodos: Pesquisa com abordagem qualitativa do tipo Estudo de Caso. Aprovada pelo Comitê de Ética em Pesquisa que envolve Seres Humanos da Faculdade de Medicina de Marília. Participaram oito docentes, por meio de entrevista e os dados foram interpretados pela Análise de Conteúdo na Modalidade Temática. Resultados: Emergiram três categorias analíticas: na Unidade de Prática Profissional (UPP) a integralidade é desenvolvida no cuidado das pessoas, famílias e comunidades, refletida junto à equipe de saúde; dificuldade de problematização para desenvolvimento da integralidade na UPP e foco na doença. Conclusão: Vislumbra-se o ensino-aprendizagem tendo em vista o cuidado na perspectiva da integralidade.

Descritores: Atenção Primária à Saúde; Integralidade em Saúde; Educação em Saúde; Gestão em Saúde; Assistência Integral à Saúde.

\section{ABSTRACT}

Objective: The present research analyzed the comprehensiveness awareness from the professors of medicine's and nursing professors' perspective involved with Primary Health Care (PHC). Methods: A qualitative case study approach was used in the research, approved by the Human Research Ethics Committee of the Faculty of Medicine of Marilia. The research participants were eight professors with whom an interview was made, being data interpreted subsequently by the Thematic Content Analysis tool. Results: Three analytical categories emerged: at the Professional Practice Unit (PPU) comprehensiveness is carried out with the care of individuals, families and communities, reflected by the healthcare team; issues formulating problematic matter to develop comprehensiveness at PPU and focus on disease. Conclusion: We long for a teaching/learning process that regards care in the comprehensiveness' perspective.

Descriptors: Primary Health Care; Comprehensiveness in Healthcare; Healthcare Education; Healthcare Management; Comprehensive Health.
Artigo Original
Elza de Fátima Ribeiro Higa ${ }^{(1)}$ Antônio Henrique Rodrigues

Passos $^{(1)}$

Márcia Aparecida Padovan

Otani $^{(1)}$

Bernardino Geraldo Alves

Souto $^{(2)}$

1) Faculdade de Medicina de Marília FAMEMA - Marília (SP) - Brasil

2) Universidade Federal de São Carlos UFSCAR - São Carlos (SP) - Brasil
Este artigo seguiu as normas e formatação estabelecidas pelo $5^{\circ}$ CIAIQ - Congresso Ibero-Americano em Investigação Qualitativa. 


\section{INTRODUÇÃO}

A Lei Orgânica de Saúde (LOS) no 8080, de 1990, do Sistema Único de Saúde (SUS) entende "integralidade de assistência como um conjunto articulado e contínuo das ações e serviços preventivos e curativos, individuais e coletivos, exigidos para cada caso em todos os níveis de complexidade do sistema"(1).

Um dos grandes desafios dos sistemas de saúde universais e integrais é a formação de recursos humanos (RH) de acordo com as necessidades de saúde da população. Desde o movimento de Renovação da APS, estimulados pela Organização Pan-americana de Saúde (OPAS) e Organização Mundial da Saúde (OMS) em 2003, os esforços que todos os países estão realizando nos processos de reforma visam fortalecer os sistemas de saúde para que atinjam os objetivos globais, regionais, nacionais e locais de saúde. Um dos elementos essenciais, que caracterizam os sistemas de saúde com base na APS é o de RH apropriados ${ }^{(2)}$.

A Constituição Federativa do Brasil explicita que compete ao SUS "ordenar a formação de recursos humanos na área de saúde" e o artigo 27 da LOS aponta os serviços de saúde como campos para o ensino e pesquisa, espaços de ensino-aprendizagem, integrando a assistência, a gestão e a formação em saúde ${ }^{(3,4)}$.

A implantação da Lei de Diretrizes e Bases (LDB) ${ }^{(5)}$ da Educação Nacional em 1996 e das Diretrizes Curriculares Nacionais $(\mathrm{DCN})^{(6)}$ para os cursos de graduação na área da saúde em 2001 induziu várias mudanças na busca de inovações curriculares para a construção de novas concepções nas instituições de ensino, particularmente a contribuição de um modelo pedagógico que considera as dimensões sociais, econômicas e culturais da população ${ }^{(7,8)}$.

Mesmo com todo o investimento dos Ministérios, Secretarias de Estado e Municípios, a ausência de um egresso formado sob as bases referenciais em concordância com o modelo de vigilância em saúde, assim como a ausência de regulação do número de especialistas em medicina de família, no caso da carreira médica, acabou se tornando um limitador na expansão das equipes, destacando o fato de que a grande maioria dos médicos inseridos na rede básica não teve treinamento específico para a complexidade da $\operatorname{APS}^{(9,10)}$.

Algumas experiências têm possibilitado aos estudantes a atuação junto às Equipes de Saúde da Família (ESF), o que lhes permite compreender os determinantes do processo saúde-doença, a importância das medidas de promoção e prevenção e da ESF como espaço de gestão do cuidado e gestão da clínica ${ }^{(11,12)}$. Alguns municípios têm amadurecido a ideia da Integração Ensino Serviço na busca de articulação num sistema mais complexo e orientado para a inovação das práticas de saúde e da formação profissional, assumindo que o processo é de construção permanente, objetivando a conformação dos projetos e a conformação de sujeitos orientados por projetos coletivos que resgatam valores como a solidariedade e a responsabilidade compartilhada, ultrapassando o conceito de parceria entre os componentes (academia, serviços, comunidade) $^{(13)}$.

$\mathrm{Na}$ ótica dos egressos de um curso de medicina, a inserção nos serviços de APS possibilitou o conhecimento da organização e do funcionamento do serviço de saúde, do contexto de vida dos usuários, além de facilitar a criação do vínculo e o desenvolvimento do raciocínio clínico. Eles destacam, no entanto, que a imaturidade do estudante nas séries iniciais impede maior aproveitamento dessa vivência. Essa percepção reforça e alinha-se às novas diretrizes curriculares para os cursos de medicina publicadas em 2014, que propõem $35 \%$ da carga horária do internato (quinta e sexta séries) desenvolvidas na APS ${ }^{(10,12,14)}$.

Nesse sentido, uma revisão de literatura ${ }^{(15)}$ sobre experiências de treinamento de alunos de medicina no SUS desenha um cenário positivo para a integração entre docentes e profissionais de saúde como um caminho da formação médica atual.

Documento realizado em conjunto pela Associação Brasileira de Educação Médica (ABEM) e Sociedade Brasileira de Medicina de Família e Comunidade (SBMFC) revela que desde 2005, estas sociedades científicas vêm empreendendo esforços conjuntos de produção técnica e pedagógica para apoiar as escolas médicas em seu processo de mudança, engajados e baseados nas $\mathrm{DCN}^{(16,17)}$.

A APS é ponto de convergência entre estas duas instituições, na medida em que as DCN apontam para uma superação do modelo biomédico para um novo paradigma da integralidade e da integração com o sistema de saúde e as necessidades de saúde da população, descentralizando o ensino da medicina dos hospitais para toda a rede de saúde e tendo apostado na ESF como forma de reorganizar a atenção à saúde no país ${ }^{(16,17)}$.

Esse novo paradigma propõe foco na saúde e não na doença, integração do ciclo básico com o clínico e aproximação da teoria com a prática, por meio da articulação entre a universidade e o sistema de saúde, em seus diferentes graus de complexidade ${ }^{(11,12)}$.

Uma proposta transformadora dos processos de formação e organização dos serviços seria alicerçada numa adequada articulação entre o sistema de saúde, suas várias esferas de gestão e as instituições formadoras. Tal proposta, conhecida como Quadrilátero da Formação, colocaria em evidência a formação para a área da saúde como construção da Educação em serviço/Educação permanente em saúde, 
agregando o desenvolvimento individual e institucional, os serviços, a atenção à saúde e o controle social ${ }^{(8)}$.

No atual contexto da saúde brasileira, há uma efervescência de movimentos voltados à construção de novos perfis de competência profissional, visando responder mais direta e prontamente às necessidades da sociedade. As instituições formadoras têm sido tensionadas a reconhecer as mudanças originadas no trabalho, como a ESF, os novos valores e demandas, como a humanização do cuidado, e a ampliar a reflexão sobre a atuação da escola, educadores, gestores e profissionais ${ }^{(18,12)}$.

A Faculdade de Medicina de Marília (Famema) desenvolve a formação profissional em consonância com as diretrizes do SUS e com as DCNs em seus cursos de Enfermagem e Medicina. Implementou o currículo tendo como eixo direcionador o desenvolvimento de competência profissional, de maneira dialógica, integrada, centrada no estudante de acordo com os princípios de metodologias ativas de aprendizagem ${ }^{(19)}$. Estas visam à identificação de necessidades de saúde do indivíduo, da família e do coletivo de pessoas, para o conhecimento da organização e gestão do trabalho em saúde e para o desenvolvimento de competência profissional em vigilância à saúde na perspectiva da integralidade do cuidado $^{(20)}$.

No modelo pedagógico proposto a este currículo, o estudante passa a ser compreendido como um sujeito ativo e participativo no processo ensino-aprendizagem, considerando o conhecimento prévio do mesmo e buscando com isso a aprendizagem significativa, por meio da açãoreflexão-ação. O papel do professor constitui-se pela mediação do processo ensino-aprendizagem, orientando e provocando dúvidas, entendendo o estudante como uma pessoa concreta, constituída por sua história de vida ${ }^{(20)}$.

Frente ao exposto, essa pesquisa surgiu da seguinte inquietação: como os docentes compreendem a integralidade dentro da APS? O pressuposto norteador é a necessidade de compreensão da Integralidade na APS, considerando sua polissemia, seu impacto e relevância na formação de futuros profissionais de saúde, e na qualidade do cuidado. Assim, o objetivo desta pesquisa foi analisar a compreensão sobre a Integralidade na perspectiva dos docentes dos Cursos de Enfermagem e Medicina, inseridos na APS do SUS, no Brasil.

\section{MÉTODOS}

Trata-se de uma pesquisa fundamentada em Estudo de Caso, com abordagem qualitativa, na qual se buscou identificar a compreensão dos docentes de uma faculdade de medicina e enfermagem do interior do estado de São Paulo sobre a integralidade no contexto da APS.

O Estudo de Caso se insere no paradigma construtivista e interpretativo ${ }^{(21)}$. É utilizado como estratégia de pesquisa em ciências sociais e destina-se à investigação em particular de um fenômeno, cujo objeto é uma unidade social, bem definida e analisada em profundidade ${ }^{(12)}$. Essa unidade pode ser, por exemplo, uma empresa, indústria, programa, uma instituição, uma turma ou uma entidade social, sendo assim adequado para investigar problemas práticos do cotidiano $^{(22)}$.

Participaram oito docentes dos cursos de Enfermagem e Medicina que atuaram nas Unidades de Prática Profissional (UPP) da primeira e segunda série no ano de 2014 inseridos na APS. Foi determinada, intencionalmente e por conveniência. A coleta de dados foi realizada por meio de entrevista com uma pergunta norteadora aberta: qual a sua compreensão sobre a integralidade no contexto da Unidade de Prática Profissional e como ela se insere na aprendizagem? As entrevistas com questões abertas realizadas nas dependências da faculdade tiveram duração máxima de dez minutos e foram gravadas e transcritas na íntegra pelo próprio pesquisador.

Para análise dos dados obtidos foi utilizada a Análise de Conteúdo na Modalidade Temática ${ }^{(23)}$. A análise de conteúdo organizou-se em três momentos: pré-análise, categorização e interpretação dos dados. Numerosos estudos, na área da pesquisa qualitativa dos serviços de saúde, propõemse a fazer uma descrição dos dados, enquanto outros, sua análise ou interpretação, porém muito poucos aprofundam o assunto, o que imprime certa confusão terminológica, conceitual e operacional ${ }^{(24)}$. Por esse motivo, torna-se complexo diferenciar até onde chega a análise dos dados em torno da perspectiva da compreensão da integralidade em relação ao momento preciso no qual se inicia a interpretação ${ }^{(24)}$.

Em cumprimento à Resolução 466/2012, do Conselho Nacional de Saúde ${ }^{(25)}$, a pesquisa foi aprovada pelo Comitê de Ética em Pesquisa Envolvendo Seres Humanos da Faculdade de Medicina de Marília, sob o número de protocolo 513.764 e da CAAE 23509213.0.0000.5413. Os participantes assinaram o Termo de Consentimento Livre Esclarecido (TCLE) antes do início da coleta de dados.

\section{RESULTADOS E DISCUSSÃO}

Da análise dos dados obtidos por meio das entrevistas emergiram três categorias analíticas demonstradas no quadro I. 
Quadro I - Categorias analíticas obtidas da análise dos dados das entrevistas com docentes. Marília, 2014

\begin{tabular}{|c|c|}
\hline Grupo & Categorias Analíticas \\
\hline \multirow{3}{*}{ Docentes } & $\begin{array}{l}\text { A - Na UPP a integralidade é } \\
\text { desenvolvida por meio do cuidado às } \\
\text { pessoas, famílias e comunidades de } \\
\text { forma reflexiva junto aos estudantes e } \\
\text { à equipe de saúde. }\end{array}$ \\
\hline & $\begin{array}{l}\text { B - Dificuldade de problematização da } \\
\text { realidade a partir da prática interfere no } \\
\text { desenvolvimento da integralidade na } \\
\text { UPP. }\end{array}$ \\
\hline & $\mathrm{C}-$ Foco na doença. \\
\hline
\end{tabular}

A - Na UPP a integralidade é desenvolvida por meio do cuidado das pessoas, famílias e comunidades de forma reflexiva junto aos estudantes e equipe de saúde.

Dois docentes apresentaram sua compreensão da integralidade na formação, destacando o processo educativo:

"Eu insisti muito na criação do vínculo [...] da atenção básica, fiz uma reunião com eles perguntando quais eram as necessidades de saúde [...] conhecendo muito a história, não do ponto de vista anamnese, história mesmo! A história de vida, história do trabalho, então eles conhecendo isso ai como trabalhou, onde trabalhou, com o que trabalhou, sofreu acidente, ficou doente no trabalho? A história da pessoa [...] então acho que isso aponta as necessidades que o paciente tem, então caminha pra integralidade". (D3)

"Inserir os estudantes e levá-los a compreender as pessoas, as famílias naquele contexto social, dá uma possibilidade muito grande de olhar pras pessoas. $O$ grande ganho é assim: proporcionar uma reflexão junto ao estudante, e também, incluir pessoas da equipe nessa reflexão." (D7)

As falas de ambas as docentes representam o valor dado à pessoa em seu contexto de vida e à maneira que se apreende esse conhecimento nos contatos frequentes e progressivos tanto com as famílias quanto com as equipes de saúde.

Nesse aspecto, estudo realizado em contexto semelhante demonstra como fortaleza das mudanças curriculares, a inserção em séries iniciais na comunidade como possibilidade de contribuir para a aprendizagem de um cuidado ampliado e integral ${ }^{(26)}$. Quando ocorreu a reformulação da proposta curricular nos cursos médico e de enfermagem, com a inserção da prática profissional, a formação em saúde na perspectiva da integralidade foi um dos eixos de mudança, destacando a importância do processo de reflexão junto aos estudantes e às equipes de saúde ${ }^{(26)}$.

Os caminhos da integralidade passam pela reflexão conjunta entre docentes, estudantes e equipes de saúde nos encontros semanais que podem ser ampliados, incorporandose novos atores, como os gestores e a comunidade, nos planos de cuidados $\operatorname{coletivos}^{(27)}$. No âmbito da atenção à saúde e da educação em saúde, o fornecimento de indicadores de avaliação em gestão e saúde coletiva a todos os atores sociais envolvidos na APS, em especial aos gestores dos serviços de saúde que recebem estes estudantes, como forma de facilitar o processo de aprendizagem, traria maior compreensão das propostas dos cursos e das expectativas e papéis dos estudantes e tornaria mais coesa sua atuação no sentido de uma formação integral ${ }^{(27)}$.

A importância da equipe multiprofissional para alcançar a integralidade possibilita uma maior troca de saberes e experiências, estabelecendo a valorização no conhecimento do trabalho do outro, otimizando ações em conjunto, possibilitando maior resolubilidade em solucionar problemas individuais e coletivos demandados pela população ${ }^{(28)}$.

B - Dificuldade de problematização da realidade a partir da prática interfere no desenvolvimento da integralidade na UPP

Essa característica do método de aprendizagem foi assim demonstrada:

"O eixo principal dessa dificuldade encontra-se no processo de capacitação dos docentes da instituição. Como ela é feita isso eu acho que é uma dificuldade que a gente tá tendo [...] os docentes ainda, mesmo com muito tempo na escola, eles têm muita dificuldade, não sei se é uma questão conceitual, do conceito, da palavra, o que é integralidade, as pessoas às vezes não conseguem levar pra frente [...] é a principal barreira". (D1)

"Agora como ela se insere é que aí que tá. Como é que a gente vai fazer essa metodologia, como vai inserir? Ai que entra o processo ensino-aprendizagem. É válido ele ter esse contato, mas até que ponto? É interessante ele ter ou não ter essa aproximação? Ele precisa ter? Esse contato é a realidade e vai ser o dia a dia dele [...] assim como fazer é que a gente tá com problema, eu acho que é importante esse contato, eu não tive, sou da metodologia tradicional, acho que é um ganho, eles acham que não vão ser médicos do posto, acham que vão ser médicos de consultório. Eles não acreditam”. (D6)

Nesses posicionamentos, acredita-se que possa existir uma fragilidade no processo de capacitação dos docentes que é a principal barreira no desenvolvimento da integralidade. A mudança de metodologia de ensino-aprendizagem tem 
permitido esse contato com a realidade da comunidade. No entanto, mesmo com o tempo que os docentes estão na escola permanece a dificuldade conceitual ou operacional de inserção da integralidade na prática profissional.

A primeira fala traz a reflexão de como o tema da integralidade tem aparecido nas discussões teóricas e práticas ao longo dos dois anos de UPP, colocando em dúvida, ainda, a amplitude do projeto de educação permanente, hoje concebido como a principal ferramenta de capacitação docente.

Segundo Paulo Freire em Pedagogia da Autonomia ${ }^{(29)}$, "faz parte das condições em que aprender criticamente é possível à pressuposição por parte dos educandos de que o educador já teve ou continua tendo experiência da produção de certos saberes e que estes não podem a eles, os educandos, ser simplesmente transferidos. Pelo contrário, nas condições de verdadeira aprendizagem, os educandos vão se transformando em reais sujeitos da construção e da reconstrução do saber ensinado, ao lado do educador, igualmente sujeito do processo"(29).

A partir dessa constatação, imagina-se que o processo de ensino-aprendizagem da integralidade do cuidado depende de um corpo teórico, ainda que existam dúvidas a respeito da palavra ou do conceito e que o cenário da UPP será campo de desenvolvimento dessa prática a depender da experiência do docente com essa temática. Estudo de revisão integrativa da literatura ${ }^{(30)}$ revela dificuldades na utilização de métodos inovadores e que estão relacionadas ao fato de estudantes e professores estarem acostumados com o método tradicional, exigindo mudança de postura de ambos para que haja adaptação aos novos métodos. Por outro lado, recente estudo realizado em contexto de mudança curricular identifica falhas por meio da avaliação do processo pedagógico em metodologia de aprendizagem ativa. O professor é desafiado a sair de sua zona de conforto para assumir um papel de mediação pedagógica, o que no mesmo estudo fica evidente quando as atividades reais e simuladas não estão articuladas embora a prática seja o eixo articulador, o ambiente simulado propicia segurança ${ }^{(31)}$.

Nesse sentido, a segunda fala aponta que existem dúvidas sobre a mudança de perfil desse profissional que irá trabalhar na APS. Surge uma dicotomia entre o trabalho médico no setor público e no setor privado, como se existissem duas formas de ser médico. Os questionamentos que se fazem sobre a validade do contato e da aproximação desses estudantes com a realidade da comunidade deixam uma lacuna a respeito de qual seria o futuro desses profissionais. Essa dúvida reforça o modelo biomédico hegemônico, que não valoriza os conhecimentos possíveis de serem aprendidos a partir da comunidade.

As falas corroboram a literatura que descreve sobre as mudanças de metodologias de ensino na área da saúde, a qual identifica como fragilidade a carência de suporte apropriado do corpo acadêmico e institucional para sua implementação(26). Desse modo, continuam existindo grandes desafios quando se alteram os padrões estabelecidos no meio acadêmico e no modo de se conduzir o ensino na prática profissional $^{(26)}$.

\section{$\mathrm{C}$ - Foco na doença}

Há essa preocupação para a formação por parte dos docentes:

"A gente educou a população para tratar a doença e não para fazer a prevenção, a promoção". (D2)

"Na prática profissional [...] É difícil, então a gente tenta, eles questionam muito isso comigo, "como eu vou ver diabetes se eu não sei o metabolismo"? " [...], porque eles não têm o alicerce, não têm a base para conseguir chegar na patologia". (D8)

O primeiro apontamento representa uma maneira de se fazer educação em saúde. A literatura traz que, na prática da formação, diante da dificuldade de se trabalhar conceitos de saúde, doença e do processo saúde doença no interior das faculdades, essas questões são abordadas nas séries iniciais em disciplinas sobre saúde e comunidade, administradas por docentes da área de saúde pública e não parecem ter impacto nas séries finais dos cursos quando o contato com os pacientes, tanto no nível ambulatorial quanto hospitalar, é maior ${ }^{(32)}$.

$\mathrm{Na}$ maioria dos currículos dos cursos de saúde, as áreas básicas estão separadas das áreas clínicas, ou seja, em primeiro lugar aprende-se o que é o normal, as estruturas celulares, suas funções e a organização em órgãos e sistemas para, num segundo momento, caracterizar as alterações dessas estruturas, suas anormalidades e desvios.

É isso que aparece na segunda fala da docente. Ela representa, com bastante ênfase, os limites existentes dentro dos processos de mudança curricular. Mesmo com o currículo integrado e com o objetivo de realizar visitas domiciliares nas séries iniciais para se identificarem necessidades de saúde, durante o contato com as famílias, surgem dificuldades dessa relação interpessoal. Na sequência o contato com as doenças crônicas existentes naquelas famílias aflora a curiosidade/necessidade de sua explicação fisiopatológica, o que finalmente, remete estudantes e alguns docentes às áreas básicas, colocando-as antes das pessoas. Esse é um paradoxo da necessidade de se conhecer primeiro a base, o alicerce, que seriam as ciências naturais em detrimento das ciências humanas.

Nesse sentido, a segunda fala reforça a primeira de que "a gente" não só educou a população, mas também tem educado os estudantes que se tornarão gestores e profissionais, numa lógica reducionista, a da doença e, 
culturalmente, quem trata a doença é o profissional médico, o que impacta no que Foucalt chamou de Biopoder ${ }^{(33)}$. A partir do século XVII, o poder político assumiu a tarefa de gerir a vida por meio da disciplina dos corpos ou dos controles reguladores das populações. Esses são os dois pólos em torno dos quais se desenvolveu a organização do poder sobre a vida: a disciplina anatomopolítica dos corpos individuais e a regulação biopolítica das populações. O nascimento da medicina social e a consequente preocupação do Estado pela Saúde Pública responderam a esse objetivo ${ }^{(34)}$.

Estudo realizado em escola de ensino fundamental com professores a respeito da promoção de saúde e de ambientes saudáveis revelou que sua percepção é muito mais influenciada pelo modelo biomédico de "eliminar alguma disfunção do organismo", "prevenir doenças" e "tratar uma moléstia" do que pela política de promoção da saúde nos espaços educacionais, o que se assemelha ao encontrado no âmbito universitário ${ }^{(35)}$.

\section{CONSIDERAÇÕES FINAIS}

Considerando o objetivo dessa pesquisa destacamos que alguns docentes compreendem e praticam a integralidade dentro do processo ensino-aprendizagem na APS enquanto outros não compreendem a mudança curricular e o perfil profissional como algo necessário para ir ao encontro das necessidades de saúde das pessoas, famílias e comunidades, no entanto, eles estão inseridos nesse processo no qual se espera a modificabilidade cognitiva.

Ao analisarmos a integralidade, como um princípio do SUS e da APS pode-se imaginar a importância da prática docente assistencial na construção do cuidado na perspectiva da integralidade e, portanto, na formação dos futuros profissionais.

Considerando a polissemia da integralidade, os resultados deste estudo também demonstram a movimentação que a academia está fazendo para alcançar esse entendimento, pois suas manifestações indicam a importância da compreensão das diversas formas de se desenvolver a integralidade para a melhoria do cuidado prestado.

A reorganização do sistema de saúde brasileiro orientada por uma APS abrangente, condutora do processo de atenção com boa integração da rede de serviços, e com orientação comunitária no enfrentamento de determinantes sociais, é uma perspectiva para a redução das desigualdades sociais e regionais no acesso aos serviços de saúde e na utilização desses, o que contribui para efetivar o direito à saúde em nosso país.

A inserção desde os primeiros anos nos cenários de práticas profissionais, responsabilizando tanto estudantes e docentes quanto equipes de saúde pelo cuidado individual e coletivo de uma população adscrita, todos esses aspectos possibilitam ampliar o escopo de seus resultados para parte da população brasileira, que passa a ter novas faculdades de medicina com inserção de acadêmicos nas comunidades, além dos diversos outros cursos de saúde que irão se inserir no SUS, desde as unidades de saúde até as casas das pessoas que necessitam de cuidados.

\section{AGRADECIMENTOS}

À toda equipe da biblioteca da Famema, em especial para a bibliotecária Cláudia.

\section{REFERENCIAS}

1. Brasil. Lei $\mathrm{n}^{\circ} 8.080$, de 19 de setembro de 1990 . Dispõe sobre as condições para a promoção, proteção e recuperação da saúde, a organização e o funcionamento dos serviços correspondentes e dá outras providências. Diário Oficial da União, Brasília (DF); 20 set 1990; Seção 1:18055.

2. Organização Pan-Americana da Saúde. "Sistemas de saúde com base na atenção primária: Estratégia para o desenvolvimento de equipes de atenção primária em saúde (APS)”. Washington (DC): OPAS; 2009.

3. Brasil. Presidência da Republica. Constituição: Republica Federativa do Brasil 1988. Brasília (DF): Centro Gráfico do Senado Federal; 1988

4. Brasil. Lei $\mathrm{n}^{0} 8.142$, de 28 de dezembro de 1990: Dispõe sobre a participação da comunidade na gestão do Sistema Único de Saúde (SUS) e sobre as transferências intergovernamentais de recursos financeiros na área da saúde e dá outras providências [Internet]. Diário Oficial da União, Brasília (DF); 30 dez 1990. [citado 21 jan 2015]. Disponível em: http:// www.planalto.gov.br/ccivil_03/Leis/L8142.htm

5. Brasil. Presidência da República. Lei n. 9.394 de 24 de dezembro de 1996. Estabelece as diretrizes e bases da educação nacional educação nacional. Diário Oficial da União, Brasília (DF); 23 dez 1996; Seção 1: 27833

6. Brasil. Ministério da Educação, Conselho Nacional de Educação, Câmara de Educação Superior. Resolução CNE/CES n. 4, de 7 novembro de 2001. Institui diretrizes curriculares nacionais do curso de graduação em medicina. Diário Oficial da União, Brasília (DF); 9 nov 2001; Seção 1:38.

7. Silva RCF, Barreto VHL, Vasconcelos EMR. Análise da capacidade de aliar ações padronizadas e singulares na APS. Rev Bras Educ Med. 2012;36(1):13-9. 
8. Ceccim RB, Feuerwerker LCM. O quadrilátero da formação para a área da saúde: ensino, gestão, atenção e controle social. Physis (Rio J). 2004;14(1):41-65.

9. Mello GA, Mattos ATR, Souto BGA, Fontanella BJB, Demarzo MMP. Médicos de família: ser ou não ser? Dilemas envolvidos na escolha desta carreira. Rev Bras Educ Méd. 2009;33(3):475-82.

10. Saldanha OMFL, Pereira ALB, Medeiros CRG, Dhein G, Koetz LCE, Schwertner SF, Ceccim RB. Clínicaescola: apoio institucional inovador às práticas de gestão e atenção na saúde como parte da integração ensino-serviço. Interface Comum Saúde Educ. 2014:18(Supl 1):1053-62.

11. Carvalho SOB, Duarte LR, Guerrero JMA. Parceria ensino e serviço em unidade básica de saúde como cenário de ensino-aprendizagem. Trab Educ Saúde. 2015;13(1):123-44.

12. Gomes TLCS, Higa EDFR, Passos AHR, Otani MAP, Souto BGA. Compreensão da integralidade sob a perspectiva de docentes inseridos na atenção primária à saúde. Atas CIAIQ2016 Investigação Qualitativa em Saúde [Internet]. 2016; Volume2:[10 p.]. Available from: http://proceedings.ciaiq.org/index.php/ciaiq2016 /article/view/777/764.

13. São José do Rio Preto (SP). Prefeitura de São José do Rio Preto. Secretaria de Saúde. Departamento de Planejamento. Gerência de Educação em Saúde. Documento orientador das práticas de integração ensino serviço e comunidade no município de São José do Rio Preto - SP [Internet]. São José do Rio Preto (SP); 2014. [citado em 25 out 2016]. Disponível em: http://gestao.saude.riopreto.sp.gov.br/transparencia/ arqu/iesc/manual_orientador.pdf

14. Ricardo MPF, Marin MJS, Otani MAP, Marin MS. Estudante de medicina na Estratégia Saúde da Família em séries iniciais: percepção dos egressos. Revista da Escola de Enfermagem da USP. 2014;48(Esp 2):17883.

15. Adler MS, Gallian DMC Formação médica e serviço único de saúde: propostas e práticas descritas na literatura especializada Rev Bras Educ Med. 2014;38(3):388-96.

16. Brasil. Ministério da Educação. Conselho Nacional de Educação. Câmara de Educação Superior. Resolução n. 3, de 20 de junho de 2014. Institui Diretrizes Curriculares Nacionais do Curso de Graduação em Medicina e dá outras providências [Internet]. Diário Oficial da União, Brasília (DF); 23 jun 2014. [citado em 13 fev 2015]. Disponível em: http://portal.mec. gov.br/index.php?option $=$ com_docman\&task $=$ doc download\&gid $=15874 \&$ Itemid $=$

17. Demarzo MMP, Almeida RCC, Marins JJN, Trindade TG, Anderson MIP, Stein AT, et al. Diretrizes para o ensino na atenção primária à saúde na graduação em medicina. Rev Bras Med Fam Comunidade. 2011;6(19):145-50.

18. Hafner MLMB, Moraes MAA, Marvulo MML, Braccialli LAD, Carvalho MHR, Gomes R. A formação médica e a clínica ampliada: resultados de uma experiência brasileira. Cienc Saúde Coletiva. 2010;15(Supl 1):1715-24.

19. Faculdade de Medicina de Marília. Caderno de avaliação: cursos de medicina e enfermagem. Marília (SP): Faculdade de Medicina de Marília; 2015.

20. Faculdade de Medicina de Marília. Projeto Pedagógico do Curso de Medicina. Marília (SP): Faculdade de Medicina de Marília; 2014.

21. Denzin NK, Lincohn YS. O planejamento da pesquisa qualitativa: teorias e abordagens. Porto Alegre (RS): Artmed; 2006.

22. Yin RK. Estudo de caso: planejamento e métodos. $3^{\mathrm{a}}$ ed. Porto Alegre (RS): Bookman; 2005.

23. Bardin, L. Análise de conteúdo. São Paulo (SP): Edições 70, 2012.

24. Mercado-Martínez FJ. O processo de análise qualitativa dos dados na investigação sobre serviços de saúde. In: Bosi MLM, Mercado-Martínez FJ, organizadores. Pesquisa qualitativa de serviços de saúde. $2^{\mathrm{a}}$ ed. Petrópolis (RJ): Vozes; 2009.

25. Brasil. Ministério da Saúde. Conselho Nacional de Saúde. Resolução n. 466, de 12 de dezembro de 2012 Aprova as diretrizes e normas regulamentadoras de pesquisas envolvendo seres humanos. Diário Oficial da União, Brasília (DF); 13 jun 2013; Seção 1:59.

26. Marin MJS, Lima EFG, Paviotti AB, Matsuyama DT, Silva LKD, Gonzalez C, Druzian S, Ilias M. Aspectos das fortalezas e fragilidades no uso das metodologias ativas de aprendizagem Rev Bras Educ Méd. 2010;34(1):13-20.

27. Higa EFR, Hafner MLMB, Tonhom SFR, Taipeiro EF, Moreira HM, Guimarães APC, Pinheiro OL. Indicadores de avaliação em gestão e saúde coletiva na formação médica. Rev Bras Educ Méd. 2013;37(1):529.

28. Soares DG, Pinheiro MCX, Queiroz DM, Soares DG. Implantação da puericultura e desafios do cuidado na 
estratégia saúde da família em um município do Estado do Ceará. Rev Bras Promoç Saúde. 2016;29(1):132-8.

29. Freire P. Pedagogia da autonomia: saberes necessários à prática educativa. $47^{\mathrm{a}} \mathrm{ed}$. Rio de Janeiro (RJ): Paz e Terra; 2013.

30. Pinto AAM, Marin MJS, Tonhom SFR, Ferreira MLSM. Métodos de ensino na graduação em enfermagem: uma revisão integrativa da literatura. Atas CIAIQ2016 Investigação Qualitativa em Educação [Internet]. 2016; Volume1:[10 p.]. Available from: http://proceedings. ciaiq.org/index.php/ciaiq2016/article/view/693

31. Higa EFR, Taipeiro EF, Carvalho MHR, Aguiar-daSilva RH, Tonhom SFR, Hafner MFBM. Avaliação do processo pedagógico em metodologia de aprendizagem ativa. Atas CIAIQ2016 Investigação Qualitativa em Educação [Internet]. 2016; Volume1:[9 p.]. Available from: http://proceedings.ciaiq.org/index.php/ ciaiq2016/article/view/610

32. Cunha GT. A Construção da clínica ampliada na atenção básica. São Paulo (SP): Hucitec; 2005.
33. Ferreira Neto JL, Kind L, Barros JS, Azevedo NS, Abrantes TM. Apontamentos sobre promoção da saúde e biopoder. Saúde Soc. 2009;18(3):456-66.

34. Junges JR. Direito à saúde, biopoder e bioética. Interface [Internet]. 2009, 13(29): 285-95 [citado em 25 out 2016]. Available from: http://www. scielo.br/scielo.php?script $=$ sci_arttext\&pid $=\mathrm{S} 1414$ 32832009000200004\&lng=en

35. Silva RD, Catrib AMF, Collares PMC, Cunha ST. Mais que educar...ações promotoras de saúde e ambientes saudáveis na percepção do professor da escola pública. Rev Bras Promoç Saúde. 2011;24(1):63-72.

\section{Endereço para correspondência:}

Thiago Luccas Correa dos Santos Gomes

Av Monte Carmelo, 800

CEP: 17.519-030 Marília - SP - Brasil

E-mail: gomesan@famema.br 